\title{
Substitution bessert körperliche Gesundheit
}

\begin{abstract}
- Aufgrund der strengen gesetzlichen Bestimmungen stehen viele Ärzte einer Betreuung von Suchtpatienten, insbesondere jenen mit Opiatabhängigkeit, kritisch gegenüber. Dabei sei die Behandlung Suchtkranker mindestens so befriedigend wie die Therapie anderer chronisch Erkrankter, erklärte Dr. Manfred Nowak, Landau.

Nowak kritisierte die eher hochschwelligen Substitutionsvorgaben, die viele in die offene Drogenszene zurückdränge. Zeitgemäß sei ein niedrigschwelliger Einstieg, wie er in der Schweiz seit langem mit Erfolg praktiziert werde.
\end{abstract}

Das Ziel der Abstinenz sei zwar nicht falsch, sollte aber eher als Langzeitziel denn als Voraussetzung für eine Behandlung verstanden werden, betonte Nowak. „Denn im Belohnungssystem des Gehirns gibt es keine Löschtas- te." Diese Sicht findet derzeit Eingang in die Therapie von Menschen mit Alkoholabhängigkeit.

\section{Eine hausärztliche Aufgabe?}

Nowak ermunterte explizit auch Hausärzte, sich in der Betreuung von in Schwerpunktpraxen stabil eingestellten Patienten zu engagieren, auch wenn der Substitutionspatient ein besonderer $\mathrm{Pa}$ tient sei - gleichzeitig bedürftig und fordernd. Und auch, wenn man neben der rein medizinischen Versorgung auch andere Aspekte wie Sozialtherapie, Einbeziehung der Familie oder juristische Beratung leisten müsse.

\section{Womit substituieren?}

Als Substitutionsmittel bei Opiatabhängigkeit werden vor allem Methadon/Levo-Methadon (z. B. Methadict $\%$ Methaliq ${ }^{\circ}$ ) und Buprenorphin (z.B. Bupre-

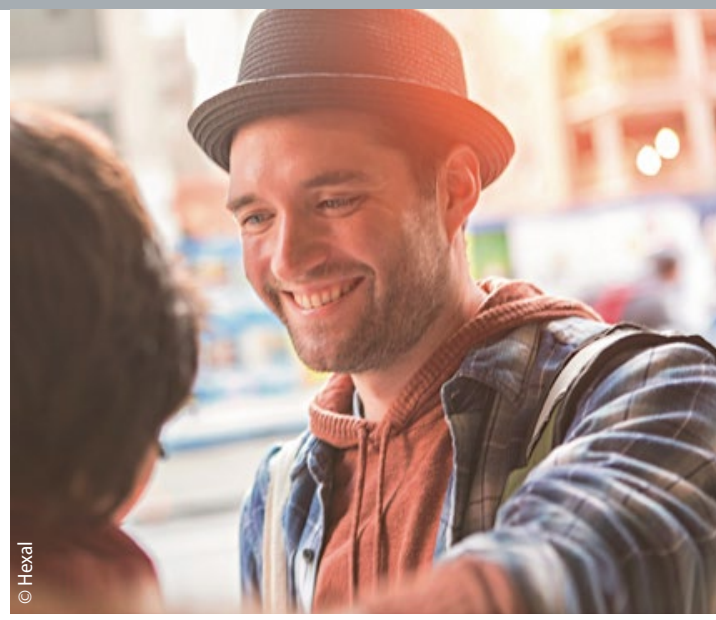

Substitution kann zurück in ein geregeltes Leben mit normalen Sozialkontakten führen.

naddict ${ }^{\circ}$ ) eingesetzt. Bei Methadict $^{\oplus}$ hob er die Darreichungsform als Tablette hervor, da sie leichter in den Urlaub mitzugeben und besser verträglich (weniger Erbrechen und Schwitzen) seien.

Wiebke Kathmann

- Meet-the Expert "Substitution - Stellenwert der medikamentengestützten Behandlung"; Landau, August 2015 (Veranstalter: Hexal)

\section{Bestimmung von Blutgerinnungsparametern in der Praxis}

\section{Laborqualität im Taschenformat}

_ Im Bereich der Primär- und Notfallversorgung sowie im Krankenhaus werden schnelle und vor allem zuverlässige Ergebnisse der Prothrombinzeit (PT) und der INR-Ergebnisse für die orale Antikoagulanzientherapie - etwa zur Reduzierung des Schlaganfallrisikos - benötigt. Diesen Anforderungen entspricht Xprecia Stride ${ }^{\circ}$. Es verwendet dasselbe Reagenz $\left(\right.$ Dade $^{\oplus}$ Innovin $^{\circ}$ ) wie die Analyse auf dem Laborsystem BCS-XP.

Seine Zuverlässigkeit bezüglich der Testergebnisse wurde unlängst in einer Studie in vier Krankenhäusern in den USA unter Beweis gestellt. Über sieben Wochen hinweg wurden 100 Patienten sowie 20 Kontrollpersonen getestet. Die von den Patienten gewonnenen Kapillar- proben wurden mit Hilfe des Xprecia Stride ${ }^{\circledR}$ vermessen, die entsprechenden Plasmaproben auf dem System Siemens BCS-XP. Die Studie zeigt eine sehr gute Korrelation $(r=0,954)$, besonders im therapeutischen Bereich von 2-4,5 INR.

Präzision und Zuverlässigkeit spielten bei der Entwicklung des Xprecia Stride ${ }^{\circ}$ eine zentrale Rolle. So verfügt jeder Teststreifen über zwei integrierte Qualitätskontrollen, durch die jede Probe nach dem Aufbringen automatisch einer Integritätsprüfung unterzogen wird. Auf diese Weise ist sichergestellt, dass nur bei positiver Kontrolle Ergebnisse vom System ausgegeben werden.

Red.

- Nach Informationen von Siemens Healthcare

\section{Kurz notiert}

Neues Target in der Therapie der Hypercholesterinämie $\rightarrow$ Nur ca $10 \%$ der deutschen Patienten mit Hypercholesterinämie erreichen mithilfe lipidsenkender Therapien den für sie empfohlenen LDL-Cholesterin-Zielwert von $<70$ $\mathrm{mg} / \mathrm{dl}$, bedauerte Prof. Elisabeth SteinhagenThiessen, Berlin. Hochrisikopatienten oder Patienten, welche die derzeitige Standardtherapie nicht vertragen, dürften künftig von PCSK9Hemmern profitieren. Das Enzym PCSK9 (Proprotein Convertase Subtilisin/Kexin Typ 9) ist wesentlich an der Regulation des LDL-Cholesterin-Spiegels im Blut beteiligt. Eine PCSK9-Inhibition beschleunigt das "Recycling“ von LDL-Rezeptoren an der Zellmembran und senkt so das LDL-Cholesterin.

Dr. Martina-Jasmin Utzt - Symposium „PCSK9-Rezeptoren zur Behandlung der Hypercholesterinämie", 121. Kongresses der Deutschen Gesellschaft für Innere Medizin; Mannheim, April 2015 (Veranstalter: Sanofi-Aventis) 